\title{
Abnormal Femoral Anteversion Is Associated With the Development of Hip Osteoarthritis: A Systematic Review and Meta-Analysis
}

\author{
Emily A. Parker, B.A., Alex M. Meyer, B.A., B.S., Momin Nasir, B.S., \\ Michael C. Willey, M.D., Timothy S. Brown, M.D., and Robert W. Westermann, M.D.
}

\begin{abstract}
Purpose: To perform a systematic review and meta-analysis of literature and to evaluate the relationship between abnormal femoral version and the development of hip osteoarthritis (OA). Methods: A systematic review was performed following Preferred Reporting Items for Systematic Reviews and Meta-Analyses (PRISMA) guidelines, evaluating Level I and II studies. Included studies had to provide granular femoral version (FV) information. The severity of OA was ranked on the Kellgren-Lawrence (KL) scale. Excel version 1808 (Microsoft, Redmond, WA) was used to perform a student $t$ test statistical analyses. Results: Our review identified 19 qualifying studies-5 Level I and 14 Level II with 1,756 patients. Patients with FV above normal range $\left(>14^{\circ}\right)$ had greater KL scores than patients with normal range FV (mean \pm standard deviation; $3.37 \pm 1.44$ vs $2.05 \pm 1.72, P<.05$ ). Analysis of KL scores in patients with FV $>24^{\circ}$ ( $>1$ standard deviation) versus patients with $\mathrm{FV}>14^{\circ}$ but $<24^{\circ}$ also demonstrated a positive correlation between increasing FV and KL $(4.00 \pm 1.96$ vs $2.34 \pm 0)$. This was significant independent of the presence or absence of developmental dysplasia of the hip. Retroverted hips $\left(\mathrm{FV}<10^{\circ}\right)$ in the present study showed variable OA results upon analysis. Conclusions: The present review suggests that elevated FV may be a risk factor for more severe hip OA with or without the presence of concurrent dysplasia of the hip. The relative amount of increased anteversion appears positively correlated with severity of OA. Although femoral retroversion may impact hip mechanics, in this review it does not appear to strongly correlate with the development of OA. Level of Evidence: II: systematic review of Level I and II studies.
\end{abstract}

$\mathbf{T}$ he hip is a mechanically complex joint, critical for gait, posture, and daily activities. The hips are a balland-socket joint, analogous to the glenohumeral joint, with multiple planes of movement. But unlike the glenohumeral joint, the hip joint is weight-bearing,

From the Department of Orthopedics and Rehabilitation, University of Iowa Hospitals and Clinics, Iowa City, Iowa, U.S.A.

The authors report the following potential conflicts of interest or sources of funding: M.C.W. reports personal fees from DePuy Synthes Sales; other from Smith $\theta$ Nephew; and personal fees from Zimmer Biomet and Stryker, outside the submitted work. R.W.W. reports personal fees, nonfinancial support, and other from Smith $\theta$ Nephew; other from Arthrex; personal fees from Medical Device Business Systems; personal fees from Linvatec; and other from Wardlow Enterprises, outside the submitted work. Full ICMJE author disclosure forms are available for this article online, as supplementary material.

Received March 23, 2021; accepted July 30, 2021.

Address correspondence to Robert W. Westermann, M.D., UIHC Sports Medicine, Attn. Hip Preservation Clinic, 2701 Prairie Meadow Dr., Iowa City, IA 52246.E-mail: Robert-Westermann@uiowa.edu

(C) 2021 THE AUTHORS. Published by Elsevier Inc. on behalf of the Arthroscopy Association of North America. This is an open access article under the CC BY-NC-ND license (http://creativecommons.org/licenses/by-nc-nd/4.0/).

2666-061X/21430

https://doi.org/10.1016/j.asmr.2021.07.029 necessitating greater osseous constraint and stability. ${ }^{1-3}$ Subsequently, abnormal mechanical stresses on the hip can lead to debilitating osteoarthritis (OA). ${ }^{2,3}$ Research of OA risk factors and prevention is multifaceted, with targets ranging from the molecular metabolism of articular cartilage to the biomechanical stresses of various sporting activities. ${ }^{4}$

Abnormalities in hip joint morphology, such as developmental dysplasia of the hip (DDH) and femoroacetabular impingement, have been shown to alter hip mechanics and promote the development of endstage hip OA. ${ }^{5}$ Femoral version, also referred to as femoral torsion, is another morphologic variable being investigated. $^{6}$ It is the angular relationship of the proximal femoral head-neck axis to the distal femoral transcondylar axis. The normal average value is between $10^{\circ}$ and $14^{\circ}\left( \pm 12^{\circ}\right)$ anteriorly, determined by anthropometric studies of individuals with no hip pathology. ${ }^{1-3,6}$ The terms "anteversion" and "retroversion" indicate a femoral neck that inclines anterior or posterior to the condylar plane, respectively. ${ }^{6}$

Screening for abnormal femoral version is common when assessing hip pain, as it has a known association 
with common hip disorders such as femoroacetabular impingement syndrome and OA. ${ }^{7,8}$ Certain aspects of the physical examination, including gait evaluation and testing for decreased passive internal rotation, are valuable tools. ${ }^{7-10}$ If the physical examination is concerning, the next step is advanced imaging, such as computed tomography (CT) or magnetic resonance imaging (MRI). ${ }^{8-11}$ Cross-sectional images from CT or MRI allow for measurement of femoral version. Threedimensional CT reconstruction images are also becoming more prevalent for assessment of hip and pelvic morphology. ${ }^{9}$ When measured by trained musculoskeletal radiologists, CT and MRI values correlate highly with one another. ${ }^{10}$

The relationship of abnormal femoral version with the development of hip OA is poorly defined. Some studies demonstrated that excess femoral anteversion increases OA risk, whereas others demonstrated significant premature OA in excess retroversion patients, and some demonstrated no significant association between OA and anteversion or retroversion OA. ${ }^{12-18}$ The limited scope and volume of research precludes conclusions about a version/OA relationship. The present review aims to comprehensively evaluate the relationship between abnormal femoral version and the development of hip OA. We hypothesized that excess femoral anteversion $\left(>14^{\circ}\right)$ contributes to anterior hip instability and therefore $\mathrm{OA}$, and that excess femoral retroversion $\left(<10^{\circ}\right)$ exacerbates impingement risk factors, also leading to hip OA.

\section{Methods}

\section{Literature Search}

Search strategies were developed with the assistance of an orthopaedic health sciences librarian with expertise in systematic reviews. Searches were developed by the authors and the librarian in July 2019 using an iterative process of gathering and evaluating terms. Comprehensive strategies, including both index and key word terms, were devised for the following databases: PubMed (including MEDLINE) and Embase (Elsevier platform). To maximize sensitivity, pre-established database filters other than the English-language filter were not used. The full PubMed search strategy below was adapted for use with the other electronic databases. Complete search strategies are available upon request. Supplementary approaches for searching included reviewing reference lists of the included studies.

In the PubMed Search strategy, MeSH terms and text words used to identify femoral version (search \#1) included ("hip joint" [MeSH:noexp]) AND ("bone malalignment" [MeSH:noexp] OR "bone retroversion"[Me SH:noexp] OR "torsion abnormality" [MeSH:noexp] OR "bone anteversion" [MeSH:noexp] OR femur* [text word] OR femor*[text word] OR acetabulofemoral[text word])
AND (version[text word] OR retroversion*[text word] OR anteversion* [text word] OR torsion[text word] OR Tönnis[text word] OR “bone malalignment*" [text word] OR retrotorsion*[text word] OR antetorsion*[text word]).

MeSH terms and text words used to identify hip OA and hip-related patient outcomes (search \#2) included (osteophytes[MeSH] OR arthritis [MeSH] OR "total hip replacement*" [MeSH] OR arthroplasty[MeSH]) AND ("patient-reported outcome*"[text word] OR "modified Harris Hip Score*" [text word] OR "chondral lesion*"[text word] OR degenerat*[text ord] OR osteophyte* [text word] OR osteoarthritis[text word] OR hemiarthroplasty*[text word] OR arthroplast*[text word] OR "THA" [text word] OR "OA" [text word] OR arthritis[text word] OR chondrosis[text word]. Our aggregate PubMed search combined these component searches (\#1 AND \#2), limited to English-language results, and resulted in a total of 2,467 papers. After we performed a similarly focused search via Embase, duplicates were removed using an approach to ensure accuracy and prevent accidental loss of records. This process was facilitated by citation-management software and supplemented by manual review of records.

Our initial search yielded 3,840 results that were evaluated according to our inclusion and exclusion criteria (Table 1). Two authors (E.P. and M.N.), with oversight from the senior authors (R.W., M.W., and T.B.), reviewed 333 full papers and screened-in 19 final studies. All were assessed quantitatively, with some also assessed qualitatively ${ }^{2,3,14,19-23}$ (Fig 1). The target independent variable was femoral version (Fig 1), which had to be provided in granular form. Studies also needed OA scores on a validated scale, or in unambiguous descriptive terms such as "normal cartilage" or "end-stage OA."

\section{Outcome Variables}

The primary outcome variable of this study was the development of hip OA. OA could be confirmed radiographically (radiographs, MRI) or during surgery. OA could also be confirmed via diagnostic indication for hemiarthroplasty or total arthroplasty of the hip, to ensure that the indication was not an excluded condition. DDH was the only permissible concurrent hip condition. All included studies had to provide OA information which was, or could be, quantified via a verified OA scoring system.

The majority of included papers used the Kellgren-Lawrence (KL) scale, widely recommended as a reliable radiologic classification for the hip. ${ }^{34}$ Therefore, for purposes of comparison, all OA data were reported as or converted to a KL score. KL scoring ranges from 0 , which represents healthy hips, to 4 , which represents end-stage OA. KL scores 1, 2, and 3 represent mild, moderate, and moderate-severe OA, 
Table 1. Inclusion and Exclusion Criteria for Femoral Version/OA Studies

\begin{tabular}{ll}
\hline \multicolumn{1}{c}{ Inclusion } & \multicolumn{1}{c}{ Exclusion } \\
\hline - English only & - Non-English studies \\
- Human only & - Animal studies \\
- Adult, adolescent, and pediatric studies & - Previous hip arthroplasty on the hip being evaluated \\
- Level I-II & - OA diagnosed clinically and not radiographically/ \\
- All years & surgically \\
- Anteversion and retroversion & Genetic abnormalities or other conditions causing hip \\
- May have: & - Slipped capital femoral epiphysis patients \\
$\begin{array}{l}\text { - History of dysplasia/developmental hip problems, FAI, or OA } \\
\text { - History of hip arthroscopy or previous hip procedures }\end{array}$ & ○ Patients with Legg-Calves-Perthes \\
- Version issues secondary to another procedure such as osteotomy or & - Conference abstracts, papers \\
$\quad$ pelvic surgery & - Meta-analyses \\
- EA/chondral defects detected incidentally during a hip procedure & - Case studies \\
progression, total hip replacements & - Technical notes
\end{tabular}

Left: A requisite set of criteria for study inclusion in the present review. Right: A list of individual criteria that resulted in exclusion of a study from the present review.

CT, computed tomography; DDH, developmental dysplasia of the hip; FAI, femoroacetabular impingement; MRI, magnetic resonance imaging; OA, osteoarthritis.

respectively. ${ }^{34}$ Score conversion was accomplished by comparing the KL score descriptions to the descriptive definitions of scores on non-KL scales and/or descriptive definitions of OA severity in the texts. This conversion was reviewed and confirmed by a senior author. Femoral version could only be provided in degrees, as "excess anteversion" and "retroversion" may have variable definitions among different authors, and cannot be quantified. ${ }^{2,3,14,16,19-33}$ If a study provided data that were further stratified, such as average version per individual OA score, this information was preferentially used for outcome variable analyses. Nonstratified data were still recorded to compile 1 of the 2 data sets for comparative analysis.

\section{Study Quality}

The mean Modified Coleman Methodology Score (MCMS) was assessed for the included studies. The average MCMS was 48.5, indicating poor quality, with standard deviation 7.5 and range from 36.60.This may be due to scores from larger hip arthroplasty studies such as Atkinson et al. (MCMS 65) 3,24,25 being negated by scores from smaller femoral version studies such as Reikeras et al. (MCMS 38). ${ }^{21,22,31}$ Low-average MCMS was anticipated for this relatively new area of research.

\section{Between-Study Heterogeneity}

Between-study heterogeneity in the present analysis was assessed via calculation of the $\mathrm{I}^{2}$ heterogeneity statistic, interpreted per ranges provided by the Cochrane Handbook for Systematic Reviews of Interventions (Version 5.0.1). ${ }^{35}$ The $\mathrm{I}^{2}$ statistic was $88.57 \%$, which "may represent substantial heterogeneity" per the corresponding Cochrane category. ${ }^{35}$ We postulated that the $\mathrm{I}^{2}$ statistic represented methodologic heterogeneity (design of studies) and employed a random effects model in anticipation of heterogeneity secondary to our inclusion/exclusion criteria. ${ }^{35,36}$ We acknowledge that the heterogeneity precludes interpretation of our findings as definitive conclusions.

\section{Statistical Analyses}

Excel version 1808 (Microsoft, Redmond, WA) was used to perform basic demographic calculations and all student $t$ tests. Student $t$ tests evaluated demographic data among all patients: mean age, mean percent of male patients, and mean body mass index (BMI). Student $t$ tests also were used to assess the study outcome variables: if excess femoral anteversion was associated with increased OA incidence and severity, and if excess femoral retroversion was associated with increased OA incidence and severity. After we discovered the highly significant association of femoral version $>24^{\circ}$ with greater KL scores, additional analyses were performed to elucidate the potential influence of concurrent DDH. These computations were limited to studies in which the average population femoral version (paFV) was greater than $24^{\circ}$. Among the paFV $>24^{\circ}$ group, the number of patients with and without DDH were compared, as was the average femoral version in each subgroup.

Because of the wide range of study population sizes, between 7 and 300 patients, all analyses of outcome variables were performed on standard error of the mean (SEM)-weighted pairs of femoral version-hip OA data. The weighted values were calculated per inverse SEM; studies with a high degree of variance therefore held less statistical significance. For comparative purposes, analyses were also completed on 2 other groups of data: population-level (nonstratified) study data weighted by SEM, and stratified SEM-weighted data only from studies that included direct analysis of a 


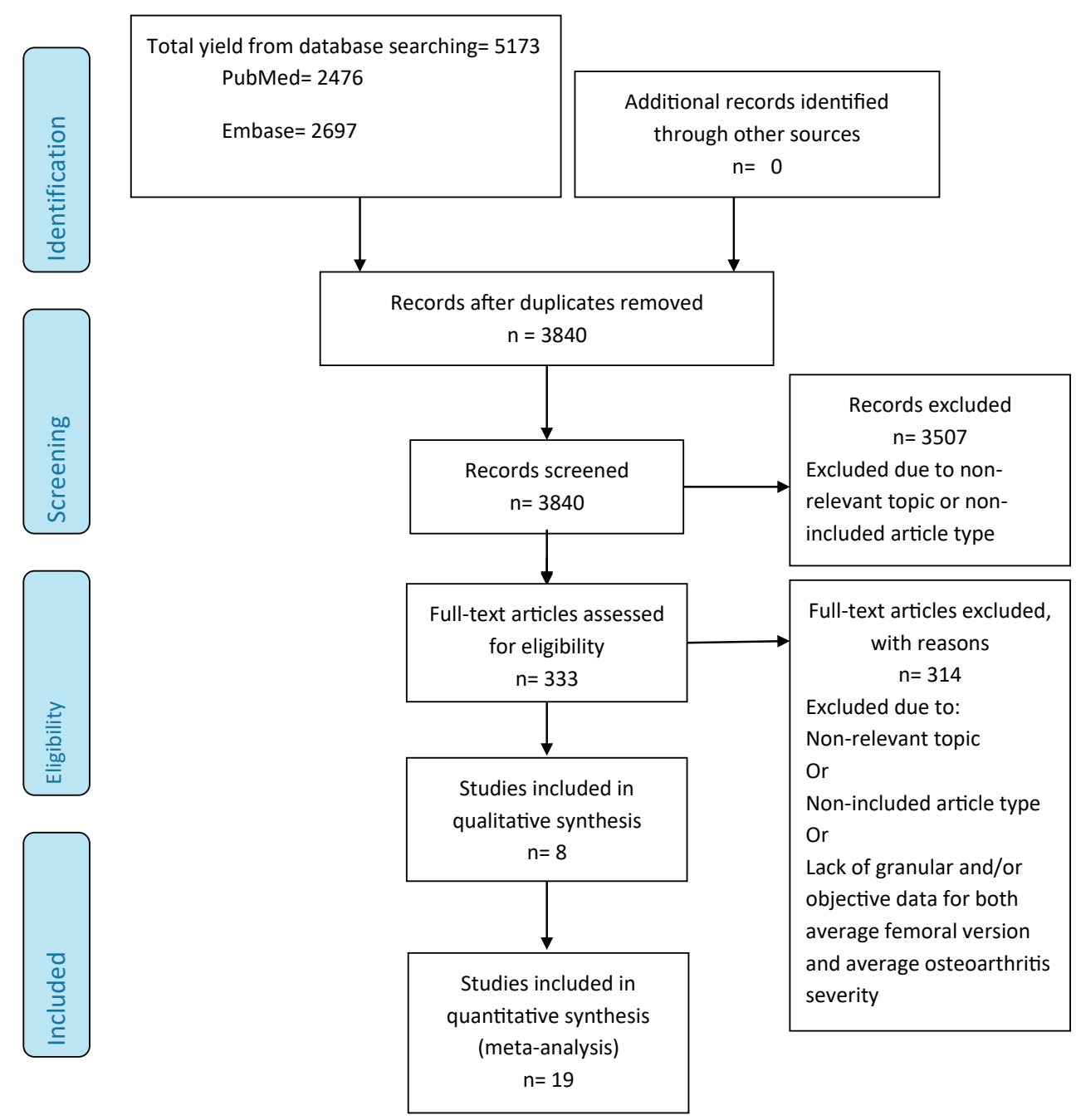

Fig 1. Preferred Reporting Items for Systematic Reviews and Meta-Analyses (PRISMA) flow diagram of included and excluded studies of femoral version and the impact on hip osteoarthritis. potential femoral version/OA relationship (qualitatively evaluated studies). This approach ensured a more accurate investigation of the true impact of femoral version from a meta-analysis level, particularly given the study size disparity present. Multifactorial analysis of the main data set showed poor fit of the femoral version/OA relationship to a linear regression; the bestfit trendline was shown to be polynomial (Fig 2 A and B).

\section{Results}

Nineteen studies including 1,756 patients met the inclusion/exclusion criteria (Table 2). The median sample size was 63, with a range spanning from 7 to 300 . The availability of other demographic data among the studies varied; 14 listed average age and sex divide of sample, ${ }^{2,3,16,19-22,24-27,29-31}$ and 6 provided average BMI. 3,19,25,26,29,30 Calculated demographic means and standard deviations showed that the average age was $60.7 \pm 6.3$ years, the average percent male was $42.4 \pm 23.3 \%$, and the average BMI was $27.05 \pm 2.30$ $\mathrm{kg} / \mathrm{m}^{2}$. In total, 15 of 19 studies measured femoral version with CT; Redmond et al. ${ }^{37}$ used MRI, Hubbard et al. ${ }^{38}$ used radiographs via the Magilligan method, and Reikeras et al. ${ }^{21}$ and Giunti et al. ${ }^{39}$ used radiographs via the Dunlap method. ${ }^{1-3,14,16,19-21,23-26,28-30,33}$

Of the 19 studies included in this review, only 8 studies directly performed computational analysis of patient femoral version values versus OA data (Table 2). 2,3,14,19-23 These studies were used for qualitative analysis and served as a comparative data group in the quantitative analyses. The other 11 included studies provided femoral version data and OA data, with no relational analysis of the 2 (Table 2). ${ }^{16,24-33}$

Qualitative review showed variable study conclusions (Table 3). 2,3,14,19-23 Argenson et al. ${ }^{19}$ and Zadeh et al. ${ }^{23}$ found a significant association between excess femoral anteversion and hip OA development, only in patients with DDH. Piazzolla et al., ${ }^{3}$ Reikeras et al., ${ }^{21}$ and Sariali et al. ${ }^{22}$ found excess femoral anteversion correlated with hip OA development. Tönnis et al. ${ }^{14}$ found an association between femoral retroversion and hip OA. 


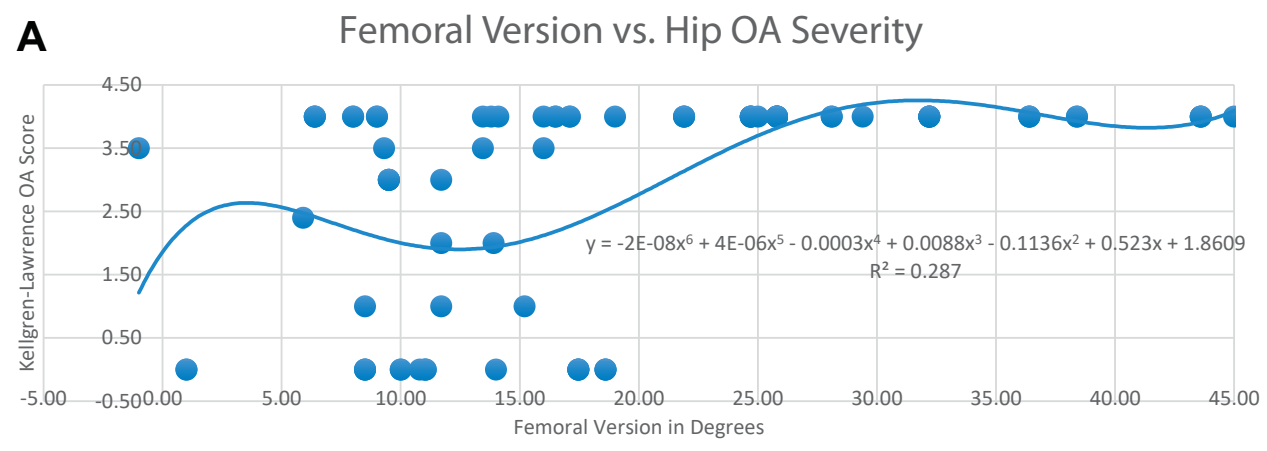

$\mathrm{OA}=$ osteoarthritis

$\mathrm{KL}=$ Kellgren-Lawrence

Fig 2. (A) Average femoral version vs. average Kellgren -Lawrence (KL) osteoarthritis scores for all studies. (B) Linear regression of average femoral version versus average KL osteoarthritis scores for all studies.

B

Femoral Version (X) Line Fit Plot

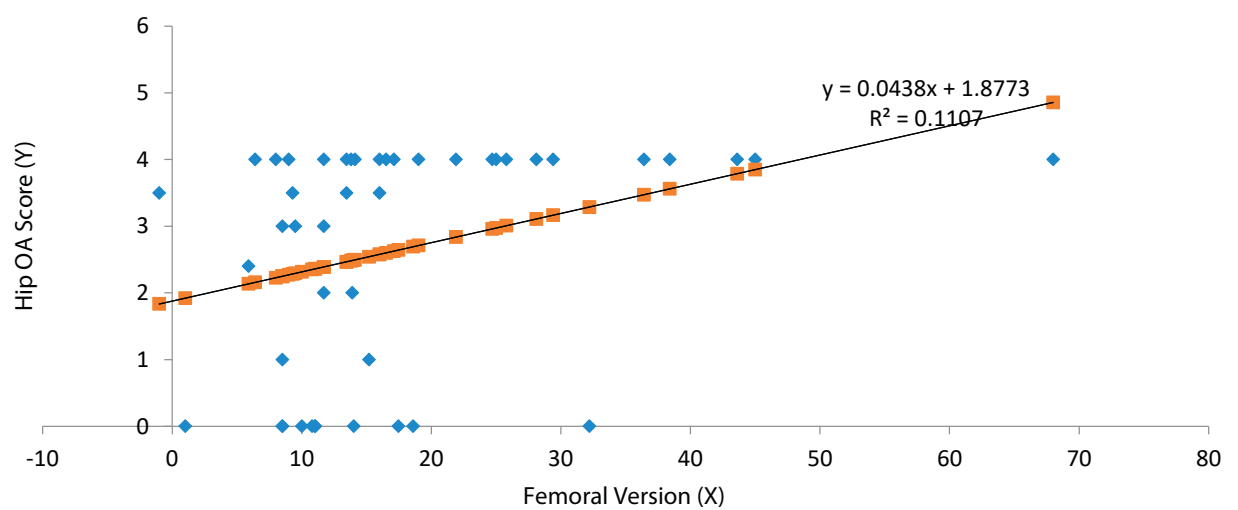

$\mathrm{OA}=$ osteoarthritis $\mathrm{KL}=$ Kellgren-Lawrence
Hayward et al. $^{2}$ and Labronici et al. ${ }^{20}$ found no association of anteversion or retroversion with the development of hip OA. The qualitative review did not lead to definitive conclusions regarding the role of femoral version in development of hip OA (Table 3).

For the first step of the quantitative analysis, performed on all 19 studies (1,756 total patients; Table 2), the relationship of excess anteversion (femoral version $>14^{\circ}$ ) and hip OA was evaluated. Patients with femoral version $>14^{\circ}$ had significantly greater KL OA scores compared with patients with normal-range femoral version (Table 4), congruent with findings of both comparative analyses (Table 4). In patients with femoral version greater than 1 standard deviation from the mean $\left(>24^{\circ}\right)$, the average KL OA scores were significantly greater compared with patients with abnormal anteversion greater than $14^{\circ}$ but less than $24^{\circ}$ (Table 4).

Given that DDH has a known association with excess anteversion, and with the development of hip OA, ${ }^{19,23}$ further analyses were performed to investigate the influence of concurrent DDH on the findings for this subgroup. These assessments involved only studies with average population femoral version greater than $24^{\circ}$, to compare the number of DDH vs non-DDH patients and the average femoral version for both patient groups $\left(\mathrm{paFV}>24^{\circ}\right)$. The analyses showed that even in the absence of concurrent DDH, femoral version $>24^{\circ}$ had a significant association with greater $\mathrm{KL}$ scores. In the femoral version $>24^{\circ}$ subgroup, $46.1 \%$ of the patients had DDH, and the average number of DDH versus non-DDH patients was not significantly different $(41.5 \pm 35.9$ vs $59.0 \pm 118.9, P=.74)$. The patients with $\mathrm{DDH}$ in these studies did have a significantly greater average femoral version $\left(29.0^{\circ} \pm 6.6^{\circ}\right.$ vs $25.0^{\circ} \pm 1.0^{\circ}$, $P<.001$ ), whereas the average KL score was 4 among both groups.

Analyses of retroversion (femoral version $<10^{\circ}$ ) and hip OA severity had inconsistent significance of results (Table 4). Analysis of our main data set and the 


\begin{tabular}{|c|c|c|c|c|c|c|c|c|}
\hline Author & $\begin{array}{l}\text { Study Level } \\
\text { of Evidence }\end{array}$ & $\begin{array}{c}\text { Patient } \mathrm{N} \\
\text { umber }(\mathrm{M} / \mathrm{F})\end{array}$ & $\begin{array}{l}\text { Mean Age } \\
\text { (Range), y }\end{array}$ & $\begin{array}{l}\text { Mean } \\
\text { Femoral } \\
\text { Version }^{\circ}\end{array}$ & Mean $\mathrm{KL}^{*}$ & $\begin{array}{l}\text { Mean } \mathrm{FV}^{\circ} \text { per Mean } \\
\mathrm{KL}^{*} \text { pairs }\left(\mathrm{FV}^{\circ}, \mathrm{KL}\right)\end{array}$ & $\begin{array}{l}\text { Direct } \mathrm{FV}^{\circ} \text {-OA } \\
\text { Analysis? }\end{array}$ & Study Summary \\
\hline Argenson et al. ${ }^{19}$ & II & $69(12 / 57)$ & $52.0(17-82)$ & 39.5 & 4 & $(36.4,4),(43.6,4),(38.4,4)$ & No $(\mathrm{N})$ & $\begin{array}{c}\text { DDH hips with OA have increased } \\
\text { femoral anteversion vs hips with OA and } \\
\text { no DDH }\end{array}$ \\
\hline Atkinson et al. ${ }^{24}$ & I & $100(61 / 39)$ & $52.8(\mathrm{~N} / \mathrm{A})$ & 8.4 & 4 & $(8.0,4),(9.0,4)$ & No $(N)$ & $\begin{array}{l}\text { No differences between sexes in } \\
\text { commonly used pelvic parameters, } \\
\text { including femoral version. }\end{array}$ \\
\hline Bargar et al. ${ }^{16}$ & I & $46(31 / 15)$ & $61.0(42-77)$ & 13.8 & 4 & $(13.8,4)$ & Yes $(\mathrm{Y})$ & $\begin{array}{l}\text { Surgeon should not use native version as } \\
\text { a guide for predicting post-THA femoral } \\
\text { version. }\end{array}$ \\
\hline Domb et al. ${ }^{25}$ & I & $175(84 / 91)$ & $57.9(33-85)$ & 6.4 & 4 & $(6.4,4)$ & No $(\mathrm{N})$ & $\begin{array}{l}\text { Robotic guidance can be used to } \\
\text { consistently correct native femoral } \\
\text { version to } 15^{\circ} \text {. }\end{array}$ \\
\hline Hayward et al. ${ }^{2}$ & II & $22(21 / 1)$ & $67.3(53-76)$ & 5.7 & 2.4 & $\begin{array}{c}(8.5,0),(9.3,3.5),(5.9,2.4) \\
(-1.0,3.5)\end{array}$ & No $(\mathrm{N})$ & $\begin{array}{l}\text { Relationship exists between anterior and } \\
\text { superior, as well as posterior and medial, } \\
\text { migration of the femoral head in OA of } \\
\text { the hip }\end{array}$ \\
\hline Hirata et al. ${ }^{26}$ & II & $122(23 / 99)$ & $65.8(42-79)$ & 28.1 & 4 & $(28.1,4)$ & No $(\mathrm{N})$ & $\begin{array}{l}\text { Greater degrees of native FV and anterior } \\
\text { stem tilt reduce discrepancy between } \\
\text { these } 2 \text { variables. }\end{array}$ \\
\hline Husman et al. ${ }^{27}$ & II & $300(171 / 129)$ & $62.0(42-76)$ & 24.7 & 4 & $(24.7,4)$ & Yes $(\mathrm{Y})$ & $\begin{array}{l}\text { Study data will contribute to } \\
\text { optimization of the design and selection } \\
\text { of femoral prostheses. }\end{array}$ \\
\hline Kudrna et al. ${ }^{28}$ & II & $150^{\dagger}$ & N/A & 22.9 & 4 & $(16.5,4),(29.4,4)$ & Yes $(\mathrm{Y})$ & $\begin{array}{l}\text { Correcting abnormal FV surgically } \\
\text { resulted in no incidences of hip } \\
\text { instability at } 1 \text {-year follow-up. }\end{array}$ \\
\hline Labronici et al. $^{20}$ & II & $42(15 / 27)$ & $69.7(42-89)$ & 11.3 & 3.4 & $\begin{array}{c}(10.8,0),(11.7,2),(11.7,3) \\
(11.7,4)\end{array}$ & Yes $(\mathrm{Y})$ & $\begin{array}{c}\text { No relationship between femoral } \\
\text { anteversion, cervicodiaphyseal angle, } \\
\text { and degree of hip OA. }\end{array}$ \\
\hline Nam et al. ${ }^{29}$ & II & $20(11 / 19)$ & $62.4(47-71)$ & 14.4 & 4 & $\begin{array}{c}(15.2,1),(13.9,2),(9.5,3) \\
(17.1,4)\end{array}$ & No $(\mathrm{N})$ & $\begin{array}{l}\text { Varus malalignment of ipsilateral knee } \\
\text { was associated with greater anteversion } \\
\text { of the femoral stem. }\end{array}$ \\
\hline Park et al. ${ }^{30}$ & II & $16(2 / 14)$ & $63.6(47-73)$ & 14.1 & 4 & $(14.1,4)$ & Yes $(\mathrm{Y})$ & $\begin{array}{l}\text { The preoperative anteversion was useful } \\
\text { for the estimation of the postoperative } \\
\text { stem anteversion. }\end{array}$ \\
\hline Piazzolla et al. ${ }^{3}$ & II & $91(34 / 57)$ & $65.8(56-78)$ & 14.7 & 4 & $\begin{array}{l}(16.0,3.5),(16.0,4),(10.0,0) \\
(13.5,3.5),(13.5,4),(11.1,0)\end{array}$ & Yes $(\mathrm{Y})$ & $\begin{array}{l}\text { A more anteverted femoral neck was } \\
\text { observed at the arthritic hip with a link } \\
\text { between FV and LBP. }\end{array}$ \\
\hline Reikeras et al. ${ }^{21}$ & II & $44(17 / 27)$ & $65.5(55-79)$ & 16.0 & 0.5 & $(14.0,0),(11.0,0),(19.0,4)$ & Yes $(\mathrm{Y})$ & $\begin{array}{c}\text { Increased femoral neck anteversion is a } \\
\text { predisposing factor for development of } \\
\text { hip OA }\end{array}$ \\
\hline Sariali et al. ${ }^{22}$ & II & $223^{\dagger}$ & N/A & 21.9 & 4 & $(21.9,4)$ & Yes $(\mathrm{Y})$ & $\begin{array}{l}\text { No correlation between femoral offset } \\
\text { and femoral anteversion. }\end{array}$ \\
\hline Soodmand et al. ${ }^{31}$ & II & $169^{\dagger}$ & N/A & 17.5 & 0 & $(17.5,0)$ & Yes $(Y)$ & $\begin{array}{c}\text { The negative correlation closest to zero } \\
\text { was femoral anteversion angle vs offset } \\
\text { angle }\end{array}$ \\
\hline
\end{tabular}




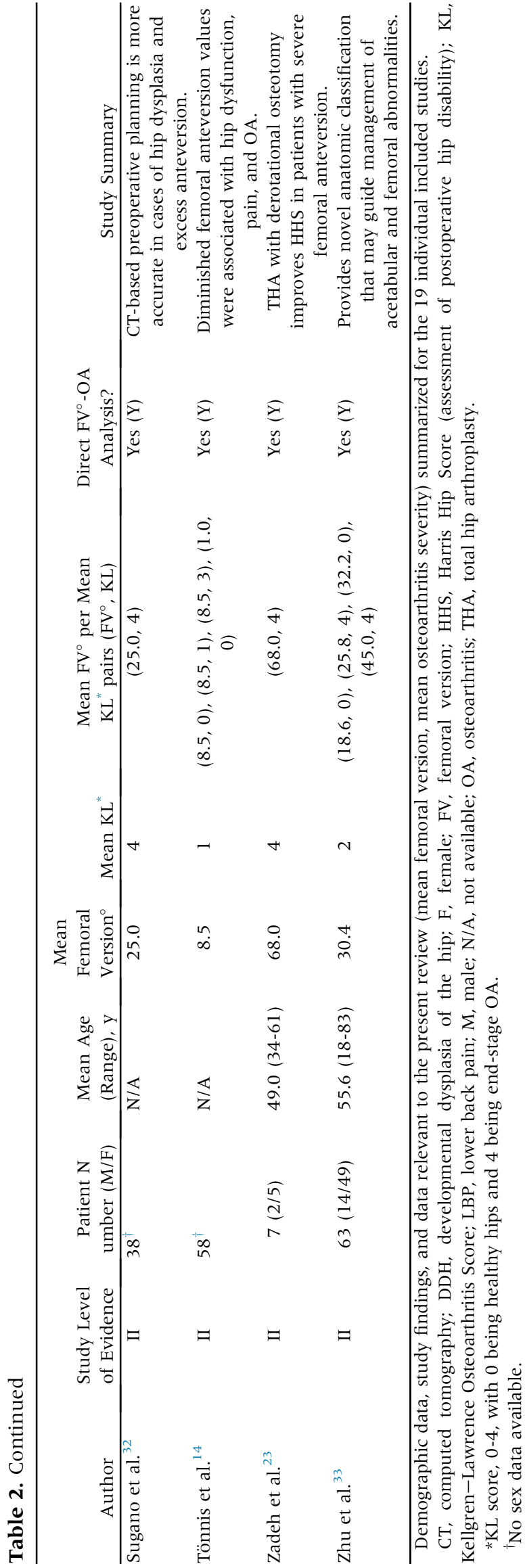

comparative analysis of the data set from studies with direct femoral version/OA analysis showed significantly lower average KL scores in patients with femoral version $<10^{\circ}$ compared with patients with femoral version $>10^{\circ}$, but no difference compared with only patients with normal-range femoral version (Table 4). In contrast, comparative analysis of nonstratified study data showed a greater average KL score in patients with femoral version $<10^{\circ}$ when compared with patients with normal range femoral version (Table 4).

KL 4 hips (end-stage OA) were shown to have significantly greater average anteversion compared to KL 1-3 hips (mild-moderate OA) $\left(25.38^{\circ} \pm 12.17^{\circ}\right.$ vs $11.34^{\circ} \pm 4.96^{\circ}, P<.05$ ) (Table 5). Results of both comparative analyses concur with this finding (Table 5). Graphing the femoral version-OA data pairs demonstrated roughly bimodal KL score increase as average femoral version values decreased or increased beyond the normal range, with the slope leveling off at the excess retroversion end of the $\mathrm{X}$-axis (Fig 2A).

\section{Discussion}

In this systematic review and meta-analysis, femoral version values above normal range $\left(10^{\circ}-14^{\circ}\right)$ showed a significant association with earlier, more severe hip OA in the main and comparative analyses. Additionally, anteversion at least 1 standard deviation from the mean $\left(>24^{\circ}\right)$ was associated with significantly greater $\mathrm{KL}$ scores compared with patients with anteversion greater than $14^{\circ}$ but less than $24^{\circ}$. Patients with KL 4 OA were shown to have significantly greater anteversion angles than patients with any other stage of hip OA (KL 1-3) in the main and comparative analyses. From a graphical standpoint, OA severity worsens in either direction as hips become increasingly anteverted or relatively retroverted. However, towards the axis end of excess retroversion $\left(<10^{\circ}\right)$ the slope starts to flatten. Statistical ambiguity regarding retroversion was clear in the results, with some showing decreased average KL scores in cases of retroversion, and others showing no impact of retroversion on average KL.

As the breadth of surgical hip procedures expands, so too does the utility of pelvis and hip anatomical measurements. Measuring femoral version began when hemiarthroplasty and total hip arthroplasty were the predominant surgical hip procedures. Studies aimed to determine if it was best to maintain native femoral version or to correct version to a more normal anatomical angle when inserting the implant. ${ }^{40-42}$ Collectively, the studies suggested correcting femoral version abnormalities during a THA.

A smaller number of femoral version studies have analyzed the impact of on younger patients with congenital hip abnormalities, finding a correlation between abnormal version and development of hip OA. Greber et al. ${ }^{43}$ found that patients with DDH were more 
Table 3. Qualitative Review of Studies Analyzing FV Versus Hip Osteoarthritis

\begin{tabular}{|c|c|c|}
\hline Author & Year & Conclusion \\
\hline Argenson et al. ${ }^{19}$ & 2005 & FV values are greater in DDH hips with OA vs hips with OA and no DDH. \\
\hline Hayward et al. ${ }^{2}$ & 1988 & $\begin{array}{l}\text { Found no correlation between osteoarthritis of the hip and abnormalities of the angle of femoral } \\
\text { anteversion. }\end{array}$ \\
\hline Piazzolla et al. ${ }^{3}$ & 2018 & $\begin{array}{l}\text { Patients with unilateral hip OA and LBP showed a marked anteverted FV in the arthritic hip and a } \\
\text { spinopelvic misalignment. }\end{array}$ \\
\hline Sariali et al. ${ }^{22}$ & 2009 & $\begin{array}{l}\text { Hips with OA had high femoral offset values and the sum of the FV and acetabular version were out of } \\
\text { the safe zone. There was no correlation between femoral offset and FV. }\end{array}$ \\
\hline Tönnis et al. ${ }^{14}$ & 1991 & $\begin{array}{l}\text { Diminished FV of the hip may cause pain and OA. Rotational osteotomies performed to restore normal } \\
\text { anteversion resulted in more relaxed and pain free hips. }\end{array}$ \\
\hline Zadeh et al. ${ }^{23}$ & 1999 & THA with derotational osteotomy improves HHS in patients with severe femoral anteversion. \\
\hline
\end{tabular}

DDH, developmental dysplasia of the hip; FV, femoral version; HHS, Harris Hip Score (assessment of postoperative hip disability); LBP, lower back pain; OA, osteoarthritis; THA, total hip arthroplasty.

likely to have increased anteversion $\left(10^{\circ}-14^{\circ}\right.$ above controls), with some measurements as high as $40^{\circ}$. Argenson et al. ${ }^{19}$ and Zadeh et al. ${ }^{23}$ also linked DDH and excess femoral anteversion, deeming to be significantly associated with hip OA. These findings reflect those of the current review; among study populations with increased OA risk due to average femoral version exceeding $24^{\circ}$, nearly one half had concurrent DDH.

Few studies have examined the correlation between femoral version and OA in patients without concomitant DDH. An early study in 1999 by Tönnis and Heinecke $^{44}$ found that decreased anteversion caused altered rotation of the hip, leading to pain $(55 / 100$ hips), altered gait patterns, and OA in some patients (11/111 hips). Decreased femoral anteversion was associated with out-toeing gait, whereas increased femoral anteversion was associated with in-toeing gait. Decreased femoral version also was associated with increased incidence of slipped capital femoral epiphysis. This research is valuable in emphasizing the many reciprocal relationships of the hip joint and factors such as femoral version. Hypothetically, abnormal version may alter gait patterns, causing excess wear and eventual OA of the knee, resulting in further compensatory movement patterns that lead to degeneration of the hip. As a "central joint," the hip is involved in countless movements of the upper and lower body during daily life.

The commonality of new research foci in the hip preservation field-DDH, FAI, version abnormalities-is that all are bony abnormalities of the hip joint. The hypothesized biomechanical consequence of bony abnormalities is altered joint movement patterns, including joint instability. This instability can accelerate the normal process of joint "wear and tear," leading to accelerated development of severe, disabling OA. Findings in current literature support this proposed pathway from bony hip abnormality, to hip instability, to hip OA. In a 2017 review of DDH contemporary concepts, Clohisy et al. ${ }^{45}$ detail the various structural factors of DDH which contribute to hip instability. A large prospective cohort study by Agricola et al. $^{46}$ established a relationship

Table 4. Osteoarthritis per Femoral Version Group, Main and Comparative Data Sets

\begin{tabular}{|c|c|c|c|c|}
\hline Data Set & $\begin{array}{l}\text { Mean Femoral } \\
\text { Version Group }\end{array}$ & $\begin{array}{l}\text { Mean KL OA } \\
\text { Grade } \pm \text { SD }\end{array}$ & $\begin{array}{l}\text { Comparison With } \\
\text { Normal FV OA }\end{array}$ & Conclusions Regarding Comparison of FV/OA Pairs \\
\hline Main & $\geq 10^{\circ}$ to $\leq 14^{\circ}$ & $1.39 \pm 1.64$ & N/A & Hips with normal range $\mathrm{FV}$, on average, have mild-moderate $\mathrm{OA}$ \\
\hline $\mathrm{n}=1,756$ & $\geq 24^{\circ}$ & $4.00 \pm 0.00$ & $P<.05$ & Excessively anteverted hips (>1 SD) have increasingly severe OA \\
\hline & $14^{\circ}<\mathrm{FV}<24^{\circ}$ & $2.34 \pm 1.96$ & N/A & $\begin{array}{l}\text { Hips with anteversion }>1 \text { SD from normal have more severe OA } \\
\text { than hips with FV exceeding normal but below l SD }\end{array}$ \\
\hline & $<10^{\circ}$ & $2.75 \pm 1.65$ & $P<.05$ & Hips with decreased anteversion have increasingly severe OA \\
\hline patients & $<10^{\circ}$ & $2.52 \pm 1.83$ & $P<.05$ & Concurs with main analysis findings \\
\hline Qualitative & $\geq 10^{\circ}$ to $\leq 14^{\circ}$ & $1.13 \pm 1.57$ & $\mathrm{~N} / \mathrm{A}$ & $\mathrm{N} / \mathrm{A}$ \\
\hline studies only & $>14^{\circ}$ & $3.97 \pm 0.13$ & $P<.05$ & Concurs with main analysis findings \\
\hline $\mathrm{n}=556$ patients & $<10^{\circ}$ & $1.16 \pm 1.55$ & $P=.48$ & Does not concur with main analysis findings \\
\hline
\end{tabular}

DDH, developmental dysplasia of the hip; FV, femoral version; HHS, Harris Hip Score (assessment of postoperative hip disability); KL, KellgrenLawrence osteoarthritis grade; LBP, lower back pain; N/A, not available; OA, osteoarthritis; SD, standard deviation; THA, total hip arthroplasty. 
Table 5. Average Femoral Version per Osteoarthritis Severity Score, Main and Comparative Data Sets

\begin{tabular}{lccc}
\hline \multicolumn{1}{c}{ Data Set } & KL 4 Average FV \pm SD & KL 1-3 Average FV \pm SD & $P$ Value \\
\hline Main & $25.38 \pm 12.17^{\circ}$ & $11.33 \pm 4.96^{\circ}$ & $<.05$ \\
Nonstratified & $26.11 \pm 12.62^{\circ}$ & $12.80 \pm 6.76^{\circ}$ & $<.05$ \\
Qualitative only & $33.26 \pm 14.36^{\circ}$ & $9.17 \pm 4.31^{\circ}$ & $<.05$ \\
\hline
\end{tabular}

FV, femoral version; KL, Kellgren-Lawrence osteoarthritis grade; SD, standard deviation.

between unstable DDH and progression to OA. Similarly, when evaluating atraumatic causes of hip instability, Shu and Safran ${ }^{47}$ discussed the impact of abnormal femoral version, which they noted to be particularly variable among the female patient population. Numerous studies in the current review conclude that abnormal femoral version, and its resulting impact on hip stability, may contribute to the development of hip OA. ${ }^{18,37,48}$

Importantly, the study by Shu and Safran ${ }^{47}$ concurs with our findings in the present study; that highly variable femoral version (i.e., $>24^{\circ}$ ) can be independently associated with hip instability and degeneration, even in the absence of other conditions such as DDH. The literature also reinforces our study finding that causes of hip instability and premature OA are much more likely, in regards to prevalence, to be dependent/additive rather than mutually exclusive individual abnormalities such as excessive anteversion. For example, a study by $\mathrm{Li}$ et al. $^{49}$ determined that among patients who all suffered from acetabular dysplasia, those who additionally had larger femoral anteversion had a significantly greater incidence of OA. This demonstrates that neither DDH nor high femoral anteversion alone caused the greater incidence of OA among study participants; rather, it was the combination of the 2 factors. ${ }^{49}$

A notable change in femoral version research is that femoral version is now being viewed as a potentially modifiable variable, in cases in which abnormal version is severe or is a notable contributing factor to abnormal structure/function of the hip. Gulan et al. ${ }^{50}$ discussed the option of femoral derotational osteotomy for young patients with femoral anteversion abnormalities and severe disruptions in function. Clohisy et al. ${ }^{45}$ documented a significant improvement in patients with periacetabular osteotomy who concurrently underwent femoral derotational osteotomy for femoral anteversion corrections. These 2 studies detail how femoral version is beginning to be tentatively analyzed as a factor that can be altered in situations where it independently impacts hip stability (excessive anteversion), or where it can be concurrently addressed with other factors detrimental to future hip health (i.e., DDH).

However, there are issues inherent to viewing abnormal femoral version as a factor which can be modified to prevent the development of hip OA. As mentioned previously, correction of abnormal femoral version is only likely to prevent hip degeneration if the version is highly abnormal and/or is identified as an additive variable leading to hip instability. The only procedure that corrects abnormal version is a femoral derotational osteotomy. Presently, it is only performed for patients with abnormal version causing significant debilitation, ${ }^{50}$ or for patients who are concurrently undergoing other hip corrections such as periacetabular osteotomy. ${ }^{45}$ A prospective multicenter study by Zaltz et al. ${ }^{51}$ noted a serious complication rate of $5.9 \%$ for experienced surgeons performing periacetabular osteotomy and associated procedures; these complications included infection, thromboembolic events, or requisite secondary surgical interventions. Buly et al. ${ }^{52}$ specifically evaluated outcomes for adult patients (mean age 29 years, range 14-59 years) undergoing derotational osteotomy for an indicated of version abnormality. Complications were similar in nature to those previously noted, but increased in number. The failure rate was $5 \%$, with only $75 \%$ of postoperative results being rated as "excellent" after recovery. In total, $78 \%$ of patients required subsequent hip procedures; mostly implant removals. ${ }^{52}$

This is one confounder when considering femoral version to be a modifiable variable: the significant increase in operative time and potential complications of associated procedures. As seen in the current review, a significant number of patients were within the normal femoral version range and had severe arthritis; a number of patients were also far outside the normal femoral version range and had healthy hips. Therefore, there is no robust evidence to suggest early surgical intervention for abnormal femoral version patients in the interest of preventing hip OA. However, that does not make femoral version a measurement without utility. Further exploration of femoral version and its interplay with other mechanical factors of the hip is indicated to determine applicability to hip injury risk stratification, as well as possible physical training interventions to counter the impact of abnormal version. Additionally, the significant association of abnormal anteversion with more severe OA, with and without concurrent $\mathrm{DDH}$, is potentially actionable.

The results of this study expand upon the previous literature evaluating OA of the hip in the context of femoral version angles, and present material for future research hypotheses in the field of hip preservation. Studies by Greber et al., ${ }^{43}$ Tönnis and Heinecke, ${ }^{53}$ and Goldstein et al. ${ }^{54}$ showed that patients with highly 
abnormal femoral version angles were more likely to develop OA. These studies also propose interactive dependent roles of femoral version in the development of OA, such as the increased likelihood of developing OA secondary to DDH with high femoral version, ${ }^{43}$ and changes in gait pattern due to abnormal version. ${ }^{54}$ Interestingly, our current findings diverge from Tönnis et al., as the patients with excess retroversion in this review had significantly lower incidence and severity of hip OA in some analyses and did not have statistically significant differences in others. With only 15 subjects, these findings are not conclusive or generalizable. They could relate to factors not obvious in the data, i.e., patients with excess retroversion having concurrent abnormalities requiring early intervention, or patients with excess retroversion being identified and studied at a younger age when degeneration may not be as pronounced.

Our study supports previous findings that abnormal femoral version often plays a role in the development of hip OA, and we determined that anteversion above the normal range does indeed have a statistically significant relationship to more severe OA scores. We also found that anteversion and OA show a "dose-dependent" relationship, with OA scores becoming significantly worse as version continued to increase above normal. These results are counter to the findings of our qualitative review, and counter to many of the study-level conclusions found in our included works. Our study shows the importance of working with larger data pools in order to accurately analyze potential statistical relationships. We also encountered variably low OA severity in femoral retroversion patients, which may warrant further investigation. Our study uses preexisting $\mathrm{OA}$ and femoral version data, provided via THA studies, to broaden our data pool, which allowed us to perform this detailed analysis. Our findings show that abnormal femoral anteversion requires numerous data points for analysis to accurately determine the role of the variable in long-term hip health.

\section{Limitations}

The predominant limitations of this review are related to heterogeneity of the included literature. Our heterogeneity statistic, $\mathrm{I}^{2}$, was $88.57 \%$ which may represent substantial heterogeneity per the Cochrane Handbook. ${ }^{35}$ As this meta-analysis used all sources with granular data, regardless of the parent study qualitative assessments of femoral version/OA, we postulate that the $\mathrm{I}^{2}$ statistic represents methodologic heterogeneity (design of studies) more than clinical heterogeneity (exposures or outcomes evaluated). ${ }^{35,36}$ Use of the KL scale was identified as a potential source of clinical heterogeneity. While reliable and valid for grading hip $\mathrm{OA}$, it primarily reflects length of time with OA rather than severity of femoral version abnormality.
Heterogeneity is also reflected in the range of MCMS scores for the included studies.

Our design to assess more subjects by including largescale studies that did not directly analyze their femoral version/OA data did substantially increase our number of data points, but was also a likely driver of our heterogeneity statistic findings. This concurs with research by Rücker et al. ${ }^{35}$ showing that $\mathrm{I}^{2}$ increases with increasing patient number of included studies. We did not believe it was appropriate to perform "one-out" post-hoc analyses in an attempt to moderate the $\mathrm{I}^{2}$ value. ${ }^{36}$ Our review did avoid some problematic components of substantial heterogeneity in reviews identified by Schroll et al., ${ }^{55}$ namely employing a random effects model and having an adequate number of included studies.

Despite the included studies being variable in design and execution, data analysis in the present study does not reveal notable disparities in the data collected. Comparative analyses with 2 differentially amassed data pools reflect an overlap of mean and standard deviation values. The strong findings related to abnormal anteversion, across the three data groups, were supported by $P<.005$ at a minimum. Nevertheless, we acknowledge that the heterogeneity positions our findings as hypothesis-generating material for future research, rather than definitive conclusions.

\section{Conclusions}

The present review suggests that elevated femoral version may be a risk factor for more severe hip OA, with or without the presence of concurrent DDH. The relative amount of increased anteversion appears positively correlated with severity of OA. While femoral retroversion may impact hip mechanics, in this review it does not appear to strongly correlate with the development of OA.

\section{References}

1. Hapa O, Yuksel HY, Muratli HH, et al. Axial plane coverage and torsion measurements in primary osteoarthritis of the hip with good frontal plane coverage and spherical femoral head. Arch Orthop Trauma Surg 2010;130:1305-1310.

2. Hayward I, Bjorkengren AG, Pathria MN, Zlatkin MB, Sartoris DJ, Resnick D. Patterns of femoral head migration in osteoarthritis of the hip: A reappraisal with CT and pathologic correlation. Radiology 1988;166:857-860.

3. Piazzolla A, Solarino G, Bizzoca D, et al. Spinopelvic parameter changes and low back pain improvement due to femoral neck anteversion in patients with severe unilateral primary hip osteoarthritis undergoing total hip replacement. Eur Spine J 2018;27:125-134.

4. Hossain M, Andrew JG. Current management of femoroacetabular impingement. Curr Orthop 2008;22:300-310.

5. Nicholls AS, Kiran A, Pollard TC, et al. The association between hip morphology parameters and nineteen-year 
risk of end-stage osteoarthritis of the hip: A nested casecontrol study. Arthritis Rheum 2011 ; 63:3392-3400.

6. Kate BR. Anteversion versus torsion of the femoral neck. Acta Anat (Basel) 1976;94:457-463.

7. Uding A, Bloom NJ, Commean PK, et al. Clinical tests to determine femoral version category in people with chronic hip joint pain and asymptomatic controls. Musculoskelet Sci Pract 2019;39:115-122.

8. Kraeutler MJ, Chadayammuri V, Garabekyan T, MeiDan O. Femoral version abnormalities significantly outweigh effect of cam impingement on hip internal rotation. J Bone Joint Surg Am 2018;100:205-210.

9. Audenaert EA, Peeters I, Vigneron L, Baelde N, Pattyn C. Hip morphological characteristics and range of internal rotation in femoroacetabular impingement. Am J Sports Med 2012;40:1329-1336.

10. Botser IB, Ozoude GC, Martin DE, Siddiqi AJ, Kuppuswami S, Domb BG. Femoral anteversion in the hip: Comparison of measurement by computed tomography, magnetic resonance imaging, and physical examination. Arthroscopy 2012;28:619-627.

11. Wells J, Nepple JJ, Crook K, et al. Femoral morphology in the dysplastic hip: Three-dimensional characterizations with CT. Clin Orthop Relat Res 2017;475:1045-1054.

12. Kraeutler MJ, Garabekyan T, Pascual-Garrido C, MeiDan O. Hip instability: A review of hip dysplasia and other contributing factors. Muscles Ligaments Tendons J 2016;6: 343-353.

13. Moya L, Buly R, Henn F, Kelly B, Ma Y, Molisani D. Femoral retroversion in patients with femoroacetabular impingement: A cofactor in the development of hip osteoarthritis. Orthop Proc 2010;92-B:526-526 (supp_IV).

14. Tönnis D, Heinecke A. Diminished femoral antetorsion syndrome: A cause of pain and osteoarthritis. J Pediatr Orthop 1991;11:419-431.

15. Westermann RW, Lynch TS, Jones MH, et al. Predictors of hip pain and function in femoroacetabular impingement: A prospective cohort analysis. Orthop J Sports Med 2017;5: $2325967117726521-2325967117726521$.

16. Bargar WL, Jamali AA, Nejad AH. Femoral anteversion in THA and its lack of correlation with native acetabular anteversion. Clin Orthop Relat Res 2010;468:527-532.

17. Kitaoka HB, Weiner DS, Cook AJ, Hoyt WA Jr, Askew MJ. Relationship between femoral anteversion and osteoarthritis of the hip. J Pediatr Orthop 1989;9: 396-404.

18. Terjesen T, Benum P, Anda S, Svenningsen S. Increased femoral anteversion and osteoarthritis of the hip joint. Acta Orthop Scand 1982;53:571-575.

19. Argenson JN, Ryembault E, Flecher X, Brassart N, Parratte S, Aubaniac JM. Three-dimensional anatomy of the hip in osteoarthritis after developmental dysplasia. J Bone Joint Surg Br 2005;87:1192-1196.

20. Labronici PJ, de Oliveira Castro GN, Neto SR, et al. Femoral anteversion and the neck-shaft angle: Relationship with hip osteoarthritis. Rev Bras Ortop 2011 ; 46: 69-74.

21. Reikeras O, Bjerkreim I, Kolbenstvedt A. Anteversion of the acetabulum and femoral neck in normals and in patients with osteoarthritis of the hip. Acta Orthop Scand 1983;54:18-23.
22. Sariali E, Mouttet A, Pasquier G, Durante E. Threedimensional hip anatomy in osteoarthritis. Analysis of the femoral offset. J Arthroplasty 2009;24:990-997.

23. Zadeh HG, Hua J, Walker PS, Muirhead-Allwood SK. Uncemented total hip arthroplasty with subtrochanteric derotational osteotomy for severe femoral anteversion. J Arthroplasty 1999;14:682-688.

24. Atkinson HD, Johal KS, Willis-Owen C, Zadow S, Oakeshott RD. Differences in hip morphology between the sexes in patients undergoing hip resurfacing. J Orthop Surg Res 2010;5:76.

25. Domb BG, Chandrasekaran S, Gui C, Yuen LC, Lodhia P, Suarez C. Can stem version consistently correct native femoral version using robotic guidance in total hip arthroplasty? Surg Technol Int 2017;31:389-395.

26. Hirata M, Nakashima Y, Itokawa T, et al. Influencing factors for the increased stem version compared to the native femur in cementless total hip arthroplasty. Int Orthop 2014;38:1341-1346.

27. Husmann O, Rubin PJ, Leyvraz PF, de Roguin B, Argenson JN. Three-dimensional morphology of the proximal femur. J Arthroplasty 1997;12:444-450.

28. Kudrna JC. Femoral version: Definition, diagnosis, and intraoperative correction with modular femoral components. Orthopedics 2005;28:s1045-s1047 (9 suppl).

29. Nam KW, Tsai TY, Dimitriou D, Li G, Kwon YM. Ipsilateral varus knee alignment correlates with increased femoral stem anteversion in primary total hip arthroplasty. Hip Int 2016;26:175-179.

30. Park KK, Tsai TY, Dimitriou D, Kwon YM. Utility of preoperative femoral neck geometry in predicting femoral stem anteversion. J Arthroplasty 2015;30:1079-1084.

31. Soodmand E, Zheng G, Steens W, Bader R, Nolte L, Kluess D. Surgically relevant morphological parameters of proximal human femur: A statistical analysis based on 3D reconstruction of CT data. Orthop Surg 2019;1 1:135-142.

32. Sugano N, Ohzono K, Nishii T, Haraguchi K, Sakai T, Ochi T. Computed-tomography-based computer preoperative planning for total hip arthroplasty. Comput Aided Surg 1998;3:320-324.

33. Zhu C, Cheng MQ, Cheng T, et al. A novel classification to guide total hip arthroplasty for adult acetabular dysplasia. Exp Ther Med 2013;6:216-222.

34. Gold GE, Cicuttini F, Crema MD, et al. OARSI clinical trials recommendations: hip imaging in clinical trials in osteoarthritis. Osteoarthritis Cartilage 2015;23:716-731.

35. Rücker G, Schwarzer G, Carpenter JR, Schumacher M. Undue reliance on $\mathrm{I}(2)$ in assessing heterogeneity may mislead. BMC Med Res Methodol 2008;8:79.

36. Patsopoulos NA, Evangelou E, Ioannidis JPA. Sensitivity of between-study heterogeneity in meta-analysis: Proposed metrics and empirical evaluation. Int J Epidemiol 2008;37:1148-1157.

37. Redmond JM, Gupta A, Dunne K, Humayun A, Yuen LC, Domb BG. What factors predict conversion to THA after arthroscopy? Clin Orthop Relat Res 2017;475:2538-2545.

38. Hubbard DD, Staheli LT, Chew DE, Mosca VS. Medial femoral torsion and osteoarthritis. J Pediatr Orthop 1988;8: 540-542.

39. Giunti A, Moroni A, Olmi R, Rimondi E, Soldati D, Vicenzi G. The importance of the angle of anteversion in 
the development of arthritis of the hip. Ital $J$ Orthop Traumatol 1985;11:23-27.

40. Herrlin K, Pettersson H, Selvik G, Lidgren L. Femoral anteversion and restricted range of motion in total hip prostheses. Acta Radiol 1988;29:551-553.

41. Worlicek M, Weber M, Craiovan B, et al. Native femoral anteversion should not be used as reference in cementless total hip arthroplasty with a straight, tapered stem: A retrospective clinical study. BMC Musculoskelet Disord 2016;17:399-399.

42. Müller M, Abdel MP, Wassilew GI, Duda G, Perka C. Do post-operative changes of neck-shaft angle and femoral component anteversion have an effect on clinical outcome following uncemented total hip arthroplasty? Bone Joint J 2015;97-B:1615-1622.

43. Greber EM, Pelt CE, Gililland JM, Anderson MB, Erickson JA, Peters CL. Challenges in total hip arthroplasty in the setting of developmental dysplasia of the hip. J Arthroplasty 2017;32:S38-S44.

44. Tönnis D, Heinecke A. Acetabular and femoral anteversion: Relationship with osteoarthritis of the hip. J Bone Joint Surg Am 1999;81:1747-1770.

45. Clohisy JC, Ackerman J, Baca G, et al. Patient-reported outcomes of periacetabular osteotomy from the prospective ANCHOR cohort study. J Bone Joint Surg Am 2017;99: 33-41.

46. Agricola R, Heijboer MP, Roze RH, et al. Pincer deformity does not lead to osteoarthritis of the hip whereas acetabular dysplasia does: Acetabular coverage and development of osteoarthritis in a nationwide prospective cohort study (CHECK). Osteoarthritis Cartilage 2013;21: 1514-1521.

47. Shu B, Safran MR. Hip instability: Anatomic and clinical considerations of traumatic and atraumatic instability. Clin Sports Med 2011;30:349-367.

48. Reikeras O, Hoiseth A. Femoral neck angles in osteoarthritis of the hip. Acta Orthop Scand 1982;53:781-784.

49. Li H, Wang Y, Oni JK, et al. The role of femoral neck anteversion in the development of osteoarthritis in dysplastic hips. Bone Joint J 2014;96-b:1586-1593.

50. Gulan G, Matovinović D, Nemec B, Rubinić D, RavlićGulan J. Femoral neck anteversion: Values, development, measurement, common problems. Coll Antropol 2000;24: 521-527.

51. Zaltz I, Baca G, Kim Y-J, et al. Complications associated with the periacetabular osteotomy: A prospective multicenter study. J Bone Joint Surg Am 2014;96:1967-1974.

52. Buly RL, Sosa BR, Poultsides LA, Caldwell E, Rozbruch SR. Femoral derotation osteotomy in adults for version abnormalities. J Am Acad Orthop Surg 2018;26: e416-e425.

53. Tönnis D, Heinecke A. Current concepts reviewacetabular and femoral anteversion: relationship with osteoarthritis of the hip*. J Bone Joint Surg Am 1999;81: 1747-1770.

54. Goldstein R, Kaye I, Slover JD, Feldman D. Hip dysplasia in the skeletally mature patient. Bull Hosp Jt Dis 2014;72:28-42.

55. Schroll JB, Moustgaard R, Gøtzsche PC. Dealing with substantial heterogeneity in Cochrane reviews. Crosssectional study. BMC Med Res Methodol 2011;11:22-22. 